\title{
Gracillin Isolated from Reineckia carnea Induces Apoptosis of A549 Cells via the Mitochondrial Pathway
}

This article was published in the following Dove Press journal: Drug Design, Development and Therapy

Background: Reineckia carnea is commonly used to treat cough, pneumonia and other diseases in China. In our previous study, it was found that the ethanol extracts of Reineckia carnea have a strong inhibitory effect on the proliferation of human lung cancer A549 cells. Here, we isolated gracillin from ethanol extracts for the first time.

Purpose: Clarify the antiproliferation effect of gracillin on A549 cells and further explore its mechanisms via the mitochondrial pathway.

Methods: Gracillin was isolated and purified by silica gel, D-101 macroporous resin and preparative RP-HPLC, then identified by NMR and HR-MS. The inhibitory effects of gracillin on the proliferation of A549 cells were detected by the MTS method. Its mechanisms were further explored by flow cytometry and Western blot.

Results: A steroid saponin, gracillin, was isolated and identified from Reineckia carnea for the first time. In a concentration-dependent and time-dependent manner, gracillin significantly inhibited the proliferation of $\mathrm{A} 549$ cells with an $\mathrm{IC}_{50}$ value at $2.54 \mu \mathrm{mol} / \mathrm{L}$ and induced morphological changes. The results of flow cytometry analysis showed that the apoptosis rate of A549 cells was significantly increased $(p<0.05)$, and the cells proportion was obviously arrested in S phase. The concentration of intracellular calcium was raised $(p<$ 0.01 ), and the mitochondrial membrane potential was greatly decreased $(p<0.01)$. In addition, the expression levels of Bax, caspase-3, cleaved caspase-3, and cytochrome $\mathrm{C}$ were dramatically up-regulated while Bcl-2 was down-regulated $(p<0.05)$ in A549 cells. Conclusion: This study confirmed that gracillin has a significant antiproliferative effect on A549 cells. Gracillin could induce the apoptosis of A549 cells through the mitochondrial pathway, which might be associated with regulation of the concentration of intracellular calcium, the mitochondrial membrane potential and the expression levels of Bax, Bcl-2, caspase-3, cleaved caspase-3, and cytochrome C.

Keywords: Reineckia carnea, gracillin, antiproliferative activity, A549 cells, apoptosis

\section{Introduction}

In recent years, lung cancer has become one of the most common malignant tumors and the leading cause of cancer death in the world. ${ }^{1,2}$ At present, the main clinical treatment for lung cancer is still surgery, radiotherapy and chemotherapy. PD-1 (anti-programmed death protein 1 antibody) showed a positive effect in treating lung cancer in clinical trials, ${ }^{3,4}$ but with deficiences in effectivity and even drug resistance, further extensive research is needed for supporting the clinical treatment of lung cancer, especially active components from natural sources.
Correspondence: Qian Liu

Gannan Medical University, \#I Yixueyuan Road, Ganzhou, Jiangxi, People's Republic of China

Tel +867978169666

Fax +867978169600

Email liuqiangmu20I7@I26.com 
Apoptosis plays an important role in the development and regulation of growth of cells. ${ }^{5,6}$ It is associated with several diseases, especially carcinomas. ${ }^{7-9}$ Inducing apoptosis of tumor cells has become a strategy in cancer treatment. Apoptosis involves a series of proteins and genes regulation, among which the mitochondrial pathway is the major one. ${ }^{10,11}$ The mitochondrial apoptosis signaling pathway is related to many proteins including antiapoptotic proteins $^{12}$ (such as Bcl-2, Bcl-x etc.) and proapoptotic proteins (such as Bax, Bak etc.). Mitochondria activate downstream apoptotic executors through releasing cytochrome $\mathrm{C}$ together with apoptosis-inducing factors. Furthermore, caspase- 3 is a critical protease which plays an important role in the molecular mechanism of apoptosis. $^{13,14}$

Reineckia carnea is a common herbal medicine widely used in southwest China including Yunnan and Guizhou provinces. The plant is domestically known as "Ji Xiang Cao", and the main active components are steroidal saponins and steroidal sapogenins. ${ }^{15}$ According to the literature, anti-tumor, anti-inflammatory, ${ }^{16}$ and anti-oxidation ${ }^{17}$ activities have been reported; specifically in our previous study, the ethanol extracts of Reineckia carnea were found to possess a strong inhibitory effect on the proliferation of human lung cancer A549 cells. ${ }^{18,19}$ Furthermore, we isolated six compounds from Reineckia carnea and a steroidal saponin, gracillin, was separated and identified from this medicinal plant for the first time. Through tests of antiproliferative activity on different tumor cell lines, we found that gracillin had the strongest inhibitory effect on A549 cells.

In addition, Min et al. ${ }^{20}$ have shown that gracillin has mitochondrial-targeted anti-tumor activity by targeting complex II. Moreover, Chen et al. ${ }^{21}$ found that gracillin could induce cell cycle arrest, oxidative stress, and apoptosis in HL60 cells, as well as significant reductions in Bcl-2 and a significant increase in Bax. In addition, $\mathrm{Hu}$ et $\mathrm{al}^{22}$ reported gracillin has cytotoxicity against human cancer cell lines from leukemia and eight solid tumor diseases. Based on previous studies, the overall potential action on cancer cells of gracillin has been widely recognized. However, the effect of gracillin on lung cancer is rarely reported and the specific mechanism is still unclear.

In this article, we explain gracillin's anti-proliferation effect on A549 cells and potential molecular mechanisms by analyzing these changes, including cell cycles, intracellular calcium levels, mitochondrial membrane potential and the expression levels of related apoptosis genes.

\section{Materials and Methods \\ Materials of Reineckia carnea}

The whole plant of Reineckia carnea was collected from Anshun of Guizhou province in China. The herb was identified as Reineckia carnea (Andrews) Kunth, by Professor Qing-wen Sun in Guizhou University of Traditional Chinese Medicine. The voucher specimen (No. 20180701) was deposited in the herbarium of the clinical medical research center of the First Affiliated Hospital of Gannan Medical University.

\section{Isolation and Characterization of Gracillin}

The dried whole plant of Reineckia carnea $(10 \mathrm{~kg})$ was powdered and extracted in $150 \mathrm{~L} \mathrm{95 \%} \mathrm{ethanol} \mathrm{under} \mathrm{reflux}$ for $2 \mathrm{~h}$. After being repeated three times, the collected extract was pooled and negative-pressure filtered, and the filtrate was evaporated to be alcohol-free under reduced pressure. The crude extract was successively extracted with petroleum ether and ethyl acetate. The ethyl acetate extract was subjected to chromatography on a silica gel column and thereafter eluted with a gradient mixture of $\mathrm{CH}_{3} \mathrm{Cl}_{3}-\mathrm{MeOH}(50: 1 \rightarrow 2: 1)$. The collected $\mathrm{CH}_{3} \mathrm{Cl}_{3}-\mathrm{MeOH}$ $(10: 1)$ fraction was further eluted with water through D-101 macroporous adsorption resin to nearly colorless, then eluted with $20 \%, 40 \%, 60 \%, 80 \%$ and $95 \%$ ethanol, successively. The acquired $60 \%$ ethanol elution fraction was purified by Sephadex LH-20 and preparative RPHPLC, and $10.12 \mathrm{mg}$ white amorphous powder was obtained. The compound is identified by Nuclear Magnetic Resonance (Bruker Avance 600, Bruker, Switzerland) and LC-TOF-MS (Triple TOFTM 5600, AB Sciex, USA).

\section{Cell Line and Culture}

A549 cells (human lung adenocarcinoma cell line) and BEAS-2B cells (human normal lung epithelial cell line) were purchased from the Chinese Academy of Sciences library (Shanghai, China). A549 cells were cultured in F-12K medium (21,127-022; Invitrogen, USA) containing $10 \%$ fetal bovine serum $(10,099,141$; Gibco, USA) and $1 \%$ streptomycin/penicillin (15,140,122; Gibco, USA). BEAS-2B cells were cultured in RPMI 1640 Medium (A1049101; Gibco, USA) containing 10\% fetal bovine serum and $1 \%$ streptomycin/penicillin. The cells were cultured at a density of $6 \times 10^{3}$ to $6 \times 10^{4}$ cells $/ \mathrm{cm}^{2}$ in a $37{ }^{\circ} \mathrm{C}$ incubator with $5 \% \mathrm{CO}_{2}$. All the cell lines were exchanged and passaged 2-3 times per week. 


\section{Cell Proliferation Analysis}

The cell proliferation was detected by means of the MTS Assay Kit (K300-500; Biovision, USA). The administration group (gracillin) and the positive control group (taxol) were respectively set at a series of concentrations $(0.25$, $0.5,1,2,4 \mu \mathrm{mol} / \mathrm{L}$ ), while the negative control group (without medication) and the blank group (no cells) were also set. The proliferation inhibition of A549 cells that was treated by gracillin for 12, 24 and $48 \mathrm{~h}$ was detected by MTS. Then each well was incubated with $20 \mu \mathrm{L}$ MTS for $3 \mathrm{~h}$ in the $37^{\circ} \mathrm{C}$ incubator. Varioskan Flash was used to detect the absorbance at $492 \mathrm{~nm}$. Gracillin and taxol with the same concentration gradient acted on BEAS-2B for 48 $\mathrm{h}$, and the proliferation effect of gracillin on BEAS-2B was detected by the same method .

\section{Cell Apoptosis Assay}

A549 cells were cultured in 6-well plates at a density of $1.5 \times 10^{5} /$ well and cultured for $12 \mathrm{~h}$, and then treated with a series of concentrations of gracillin $(0.25,0.5,1,2,4$ $\mu \mathrm{mol} / \mathrm{L}$ ) for $24 \mathrm{~h}$. The cells were stained with Hoechst 33,258 staining buffer (KGA211; Nanjing KeyGen Biotech, China) and the morphology of apoptosis was observed by fluorescence microscopy. The apoptotic rate of cells was detected by an annexin V-fluorescein isothiocyanate (FITC)/Propidium iodide (PI) apoptosis kit (556,547; BD Pharmingen, USA). The cells of each well were separately collected and added to $5 \mu \mathrm{L}$ of FITC Annexin V and $5 \mu \mathrm{L}$ PI, then incubated for $15 \mathrm{~min}$ in dark. The rate of apoptotic cells (in the FITC+/PI- and FITC+/PI+ quadrant) was measured by flow cytometry.

\section{Cell Cycle Analysis}

PI stained DNA was measured by flow cytometry to reveal the cell cycle. After being treated with various concentrations of gracillin for $24 \mathrm{~h}$, the cells of each well were separately collected and fixed with $70 \%$ ethanol at $4{ }^{\circ} \mathrm{C}$ overnight. Each group of cells was dyed in dark conditions with a $300 \mu \mathrm{L} \mathrm{PI/RNase} \mathrm{stain} \mathrm{buffer} \mathrm{for} 15 \mathrm{~min}$, and the distribution of cell cycles was then detected using a flow cytometer.

\section{Assessment of Intracellular Calcium Concentration}

The intracellular calcium concentration was measured with a Fluo-8 No Wash Calcium Assay Kit (36,315; AAT Bioquest, USA). After the treatment of cells for $24 \mathrm{~h}$, each group of cells was separately collected and added to $5 \mu \mathrm{mol} / \mathrm{L}$ of FLUO- $8 \mathrm{AM}$ and incubated at $37{ }^{\circ} \mathrm{C}$ for 30 min. The average fluorescence intensity of the cells was measured by flow cytometry.

\section{Assessment of Mitochondrial Membrane Potential}

The mitochondrial film potential was detected using the A JC-10 mitochondrial film potential determination kit (22,801; AAT Bioquest, USA). The cells of each well were separately collected and added to $500 \mu \mathrm{L}$ of JC- 10 dye-loading solution and incubated at $37{ }^{\circ} \mathrm{C}$ for $20 \mathrm{~min}$. The red fluorescence intensity and green fluorescence intensity of the cells were measured by flow cytometry. The mitochondrial membrane potential of the cells was expressed as the ratio of the intensity of red fluorescence to the intensity of green fluorescence.

\section{Real-Time Quantitative PCR}

The mRNA expression levels of Bcl-2, Bax and caspase-3 were measured by qPCR. The internal control is $\beta$-actin. Total RNA from the cells was extracted with TRIzol lysate (15,596,026; ambion, USA). Reverse transcription of RNA was carried out by a first-strand cDNA synthesis kit (K1622; Thermo Fisher Scientific, USA). Then, select the main hybrid kit $(4,472,908$; Thermo Fisher Scientific, USA) for qPCR processing according to SYBR. The qPCR included denaturation $\left(50{ }^{\circ} \mathrm{C}\right.$ for 2 min and $95{ }^{\circ} \mathrm{C}$ for $2 \mathrm{~min}$ ) and 40 cycles of amplification $\left(95{ }^{\circ} \mathrm{C}\right.$ for $15 \mathrm{~s}$ and $65{ }^{\circ} \mathrm{C}$ for $1 \mathrm{~min}$ ). The PCR products were examined by electrophoresis with $2 \%$ agarose gel. The primers were as follows: $\beta$-actin (forward: CCTGGCACCCAGCACAAT; reverse: GGGCCGGACT CGTCATAC), Bax (forward: GATGCGTCCACCAAGA AGCTGAG; reverse: CACGGCGGCAATCATCCTC TG), Bcl-2 (forward: TGCCACCTGTGGTCCACCTG; reverse: TGGCTGGACATCTCGGCGAAG) and caspase-3 (forward: TGGAAGCGAATCAATGGACTCTGG; reverse: CCAGACCGAGATGTCATTCCAGTG).

\section{Western Blot Assay}

The protein expression of Bcl-2, Bax, caspase-3 and cytochrome $\mathrm{C}$ was measured by Western blot. The internal control was $\beta$-actin. After the cells were treated with gracillin for $24 \mathrm{~h}$, the total protein of each group was extracted by a RIPA buffer (R0010; Solarbio, China) with 1\% phenylmethylsulfonyl fluoride (PMSF). Then the concentration of the protein was 
detected by a BCA reagent kit (P1511; Applygen, China). The proteins were separated by $12 \%$ SDS-polyacrylamide gel electrophoresis and electro-transferred onto a polyvinylidene fluoride membrane. The membrane was blocked in 5\% nonfat dry milk (prepared by $1 \times$ Tris-buffer saline and $0.05 \%$ Tween $20 \%$ ) for $2 \mathrm{~h}$ and then incubated with the primary antibody (Applygen, Beijing, China) for $12 \mathrm{~h}$ at $4{ }^{\circ} \mathrm{C}$. The membrane was rinsed and incubated at room temperature for $2 \mathrm{~h}$ with Horizontal shaker (Applygen, Beijing, China). Finally, the membrane was rinsed again and incubated with the chemically luminescent solution for exposure. Protein intensity was measured using Image Lab software.

\section{Statistical Analysis}

Statistical analysis of the data was conducted using GraphPad Prism 7 software (San Diego 92,108, CA, USA). Data were presented in the form of mean \pm SD (standard deviation). For the measurement data that conformed to the normal distribution, Independent sample t-tests were employed for comparison between the two groups, and one-factor ANOVA tests were used for comparison between the multiple groups. $p<0.05$ was considered statistically significant.

\section{Results}

\section{Identification of Gracillin}

The structure of the compound isolated from Reineckia carnea was elucidated based on both MS and NMR spectral data: molecular formula, $\mathrm{C}_{45} \mathrm{H}_{72} \mathrm{O}_{17}$; HR-ESI-MS m/z: $885.48[\mathrm{M}+\mathrm{H}]^{+}, 723.43[\mathrm{M}+\mathrm{H}-\mathrm{Glc}]^{+}, 577.37[\mathrm{M}+\mathrm{H}-\mathrm{Glc}-\mathrm{Rha}]^{+}$
,415.32[M+H-2Glc-Rha] ${ }^{+;}{ }^{1} \mathrm{H}-\mathrm{NMR}\left(600 \mathrm{MHz}, \mathrm{CD}_{3} \mathrm{OD}\right) \delta$ : $5.20(1 \mathrm{H}, \mathrm{d}, \mathrm{J}=1.7 \mathrm{~Hz}), 4.83(1 \mathrm{H}, \mathrm{d}, \mathrm{J}=1.9 \mathrm{~Hz}), 1.05(3 \mathrm{H}, \mathrm{s}$, H-19), 0.81 (3H, s, H-18), 0.96 (3H, d, J = 6.6 Hz, H-21), $0.79(3 \mathrm{H}, \mathrm{d}, \mathrm{J}=6.6 \mathrm{~Hz}, \mathrm{H}-27), 1.25\left(3 \mathrm{H}, \mathrm{d}, \mathrm{J}=6.2 \mathrm{~Hz}, \mathrm{CH}_{3}-\right.$ Rha). ${ }^{13} \mathrm{C}-\mathrm{NMR}\left(150 \mathrm{MHz}, \mathrm{CD}_{3} \mathrm{OD}\right) \delta: 37.2$ (C-1), 29.4 (C-2), 78.6 (C-3), 38.1 (C-4), 140.5 (C-5), 121.3 (C-6), 31.3 (C-7), 31.4 (C-8), 50.3 (C-9), 36.7 (C-10), 20.6 (C-11), 39.5 (C-12), 40.0 (C-13), 56.4 (C-14), 31.8 (C-15), 80.8 (C-16), 62.4 (C-17), 16.5 (C-18), 18.4 (C-19), 41.5 (C-20), 16.1 (C-21), 109.2 (C-22), 31.0 (C-23), 28.5 (C-24), 30.1 (C-25), 66.5 (C-26), 16.6 (C-27), Glc (inner): $100.9\left(\mathrm{C}-1^{\prime}\right), 77.9$ (C-2'), 99.1 (C-3'), 69.3 (C-4'), 77.8 (C-5'), $62.4\left(\mathrm{C}-6{ }^{\prime}\right)$, Rha $(1 \rightarrow 2)$ : 101.6 (C-1”), 72.6 (C-2”), 72.3

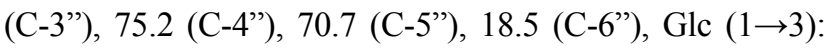
101.6 (C-1"'), 76.7 (C-2'"'), 77.9 (C-3"'), 70.8 (C-4'"), 77.9 $\left(\mathrm{C}-5^{\prime \prime \prime}\right), 60.5\left(\mathrm{C}-6^{\prime \prime \prime}\right)$. These results indicated that the compound is diosgenin $3-\mathrm{O}-\{\alpha$-L-rhamnopyranosyl- $(1 \rightarrow 2)-[\beta$ D-glucopyranosyl- $(1 \rightarrow 3)]-\beta$-D-glucopyranoside $\}$ (gracillin, Figure 1) which has been isolated previously from Dioscorea nipponica Makino, ${ }^{23}$ and it was isolated from Reineckia carnea for the first time.

\section{Antiproliferative Activity of Gracillin on A549 Cells}

The proliferation effect of gracillin was tested on A549 and BEAS-2B cells. While the cellular vitality was detected by MTS after culturing gracillin on A549 cells for 12, 24 and 48 $\mathrm{h}$, and BEAS-2B cells for $48 \mathrm{~h}$, respectively. The results showed that gracillin inhibited proliferation of the A549 cell in a concentration and time-dependent manner (Figure 2A).

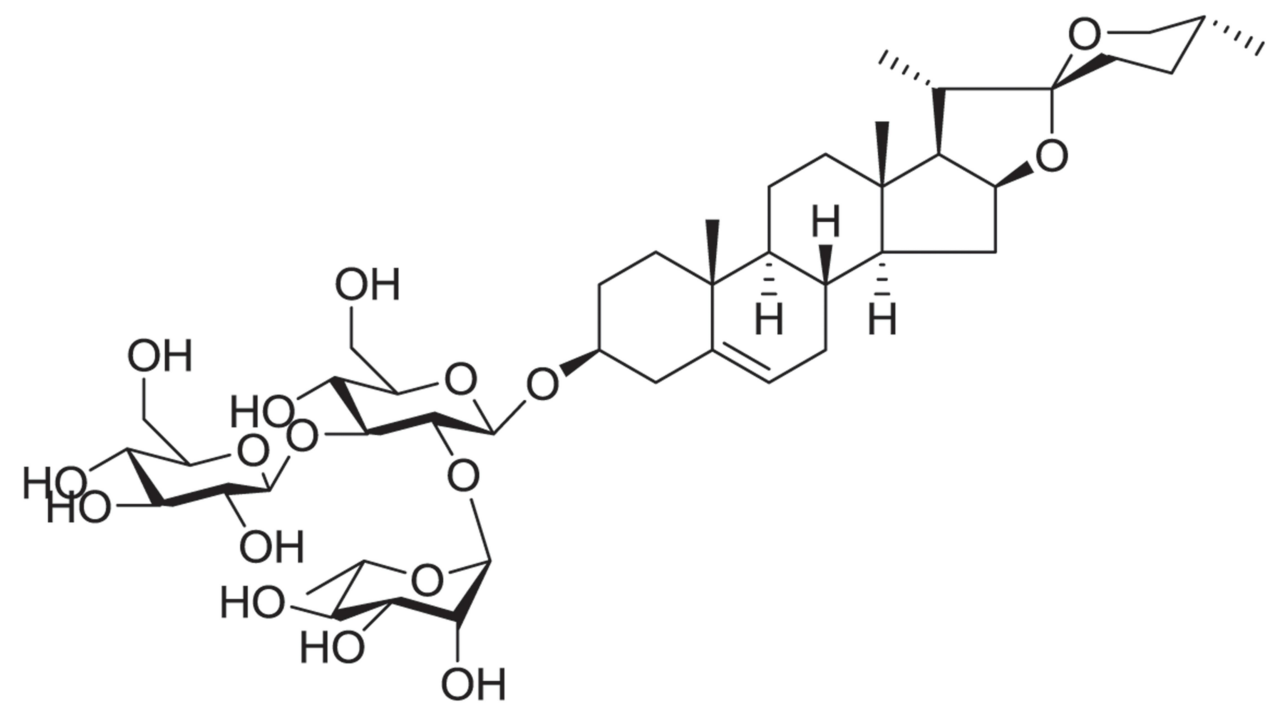

Figure I The chemical structure of gracillin. 
Positive control (taxol) showed time dependence on A549 cells (Figure 2B). After being treated with gracillin and taxol for $24 \mathrm{~h}$, the $\mathrm{IC}_{50}$ values were calculated at $2.54 \mu \mathrm{mol} / \mathrm{L}$ and $0.47 \mu \mathrm{mol} / \mathrm{L}$, respectively. At the same time, we found that the cell viability of BEAS-2B cells treated with gracillin for $48 \mathrm{~h}$ was almost unaffected compared with the control (Figure 2C).

\section{Gracillin Induced Apoptosis in A549 Cells}

In this study, we have investigated the effect of gracillin on the morphology and rate of apoptosis in A549 cells. Firstly, it can be observed that gracillin induced morphological changes in apoptosis of A549 cells by fluorescence microscopy after staining with Hoechst33258. Compared with the blank group, the gracillin treatment group showed the typical morphological characteristics of apoptotic cells, specifically, the number of cells was relatively reduced, the nucleus was pyknotic, the chromatin condensed into lumps under the nuclear membrane, and showed bright blue fluorescence staining (Figure 3A, $400 \times$ ). As a result, the apoptosis rates $(\%)$ of A549 cells in each group were $3.8 \pm 0.638,6.56 \pm 0.485,15.76$ $\pm 3.073,34.897 \pm 11.215,48.547 \pm 7.285$ and $67.43 \pm 1.997$, respectively, which showed a significant upward trend with the increase of gracillin concentration $(p<0.05, t$-test, Figure 3B).

\section{The Effect of Gracillin on the Cycle of A549 Cells}

A549 cells were treated with different concentrations of gracillin for $24 \mathrm{~h}$, and the distribution of cell cycle (G1, G2 and S phase) was detected by flow cytometry after PI/RNase staining. It was shown that when the concentration of gracillin was $1 \mu \mathrm{mol} / \mathrm{L}, 2 \mu \mathrm{mol} / \mathrm{L}$ and $4 \mu \mathrm{mol} / \mathrm{L}$, the cell ratio in $\mathrm{G} 1$ increased significantly; on the contrary, Phase $\mathrm{S}$ decreased significantly ( $p<0.05, t$-test, Figure 4$)$, while in G2 phase there was no significant difference between each group $(p>$ 0.05 , ANOVA test, Figure 4). It was suggested that gracillin may block A549 cell cycle in G1 phase and induce apoptosis.

\section{Gracillin Increased the Level of Intracellular Calcium in A549 Cells}

TheFlow cytometry was employed to evaluate the level of intracellular calcium in A549 cells after treatment with gracillin for $24 \mathrm{~h}$. The relative mean values of intracellular calcium levels (presented as average fluorescence intensity) were $2.336 \pm 0.3636,3.33 \pm 0.5503,4.487 \pm 1.047,5.873 \pm$ 0.6892 and $9.533 \pm 1.923$, respectively in the five concentrations $(0.25,0.5,1,2,4 \mu \mathrm{mol} / \mathrm{L})$ of gracillin treatment groups, which showed a significant increase compared with the untreated control group ( $p<0.01, t$-test, Figure $5)$. It can be seen that gracillin promoted the accumulation of intracellular calcium in A549 cells and showed a rising trend accompanied with the increase of gracillin concentration.

\section{The Effect of Gracillin on Mitochondrial Membrane Potential of A549 Cells}

A549 cells were treated with gradient concentrations $(0.25,0.5,1,2,4 \mu \mathrm{mol} / \mathrm{L})$ of gracillin for $24 \mathrm{~h}$, and the mitochondrial membrane potential was detected by labeling with JC-10. The result acquired by flow cytometry showed that the red and green fluorescence intensity ratio in each group was $6.81 \pm 0.529,2.01 \pm 0.229,1.21 \pm$ $0.226,0.274 \pm 0.101,0.219 \pm 0.067$ and $0.089 \pm 0.019$, respectively. Compared with the blank group, the mean value of the gracillin treatment group decreased markedly and presented in inverse proportion to the increase of gracillin concentration ( $p<0.01, t$-test, Figure 6$)$. It was indicated that the mitochondrial membrane potential of
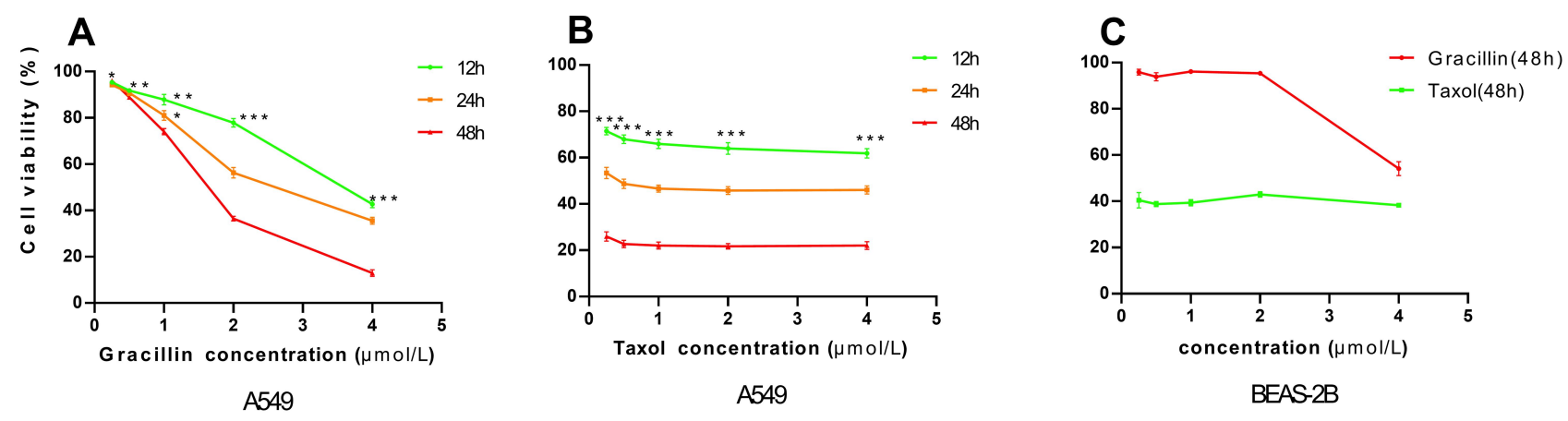

Figure 2 (A and B) The cell viability of A549 cells under the treatment of gracillin and taxol at different concentration for 12,24 and 48 hours, respectively. (C) The cell viability of human normal lung epithelial cell line BEAS-2B under the treatment of gracillin and taxol at different concentration for 48 hours, respectively. Data was expressed as mean $\pm \mathrm{SD}$. $* p<0.05, * * p<0.01$, $* * * p<0.001$, versus the untreated control group. 
A

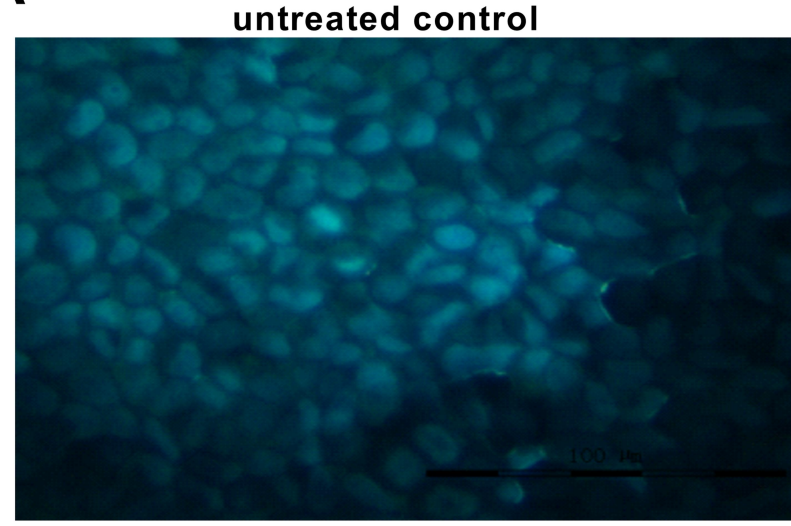

$2 \mu \mathrm{mol} / \mathrm{L}$ Gracillin

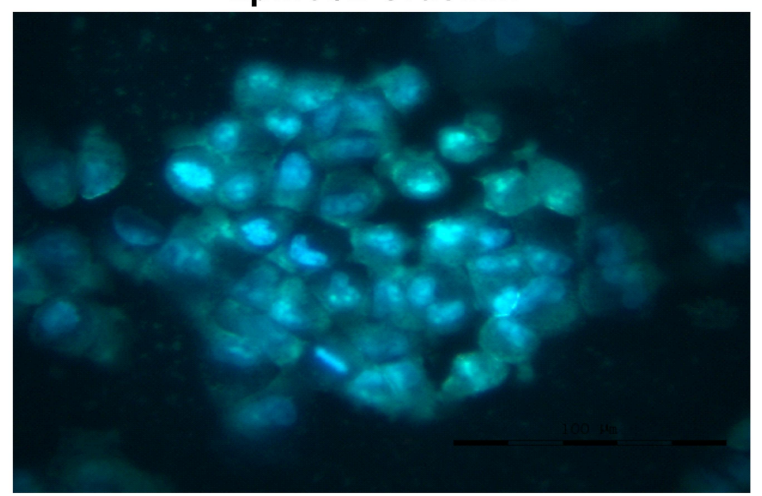

\section{B}
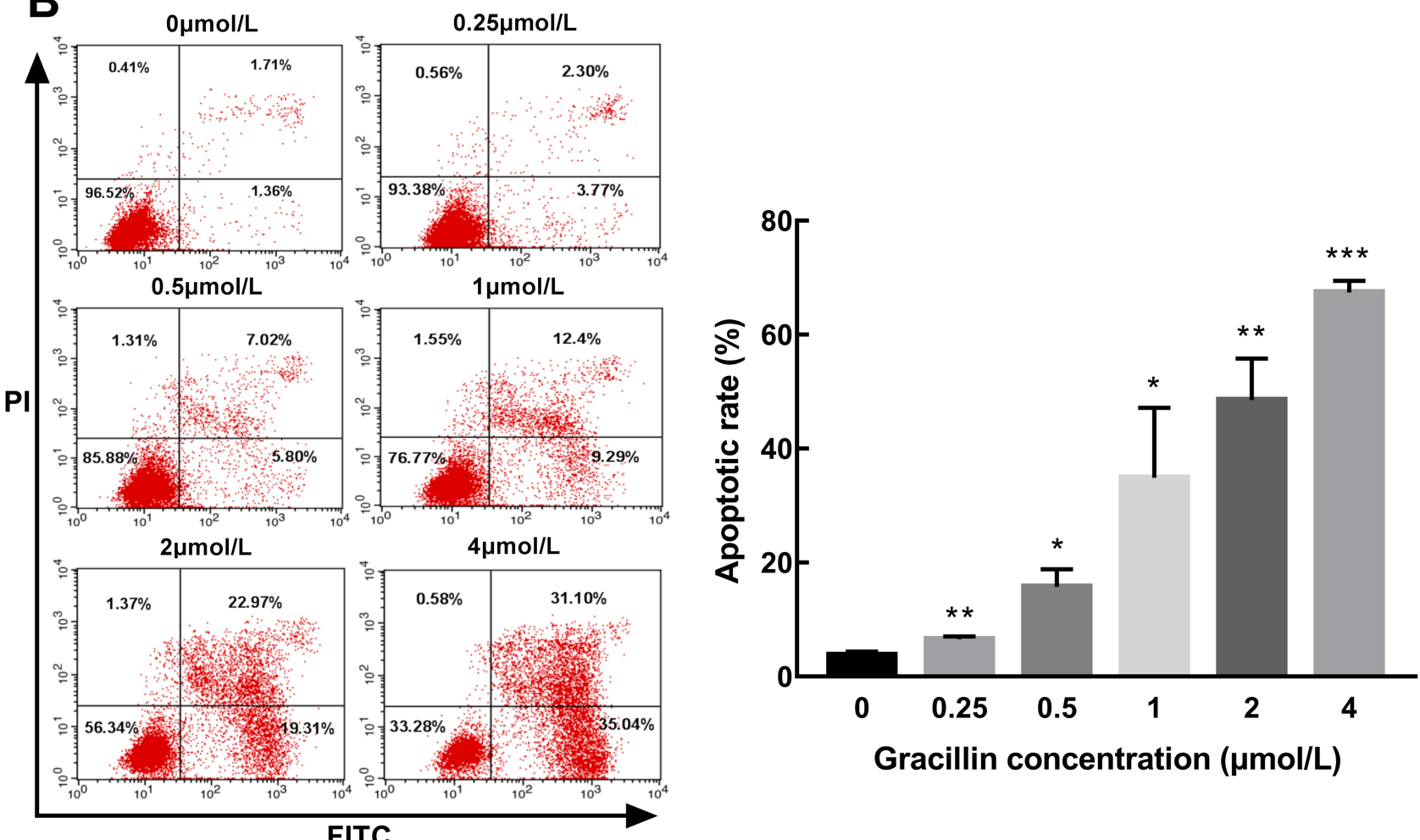

FITC

Figure 3 The effect of gracillin on apoptosis of A549 cells. (A) The morphology of apoptosis induced by gracillin in A549 cells was observed by fluorescence microscopy $(400 \times)$. (B) The percentage of apoptosis induced by gracillin was detected by Annexin V-FITC/PI double labeling. The result of flow cytometry was on the left and the corresponding statistical chart was on the right. The apoptotic A549 cells were in the FITC+/PI- and FITC+/PI+ quadrant. Data were expressed as mean \pm SD. T-test was used for the statistical analysis. ${ }^{*} p<0.05, *_{p}^{*}<0.01, * * * p<0.001$, versus the untreated control group.

A549 cells was obviously decreased after gracillin treatment.

\section{The Effect of Gracillin on}

\section{Apoptosis-Related Genes and Proteins} Expression in A549 Cells

After being treated by five concentrations $(0.25,0.5,1,2$, $4 \mu \mathrm{mol} / \mathrm{L}$ ) of gracillin for $24 \mathrm{~h}$, the qPCR result of A549 cells showed that the mRNA expression of Bax and caspase-3 were obviously up-regulated and the expression of Bcl-2 was significantly down-regulated ( $p<0.05, t$-test, Figure 7). The result of Western blot consistently showed that the expression levels of pro-apoptotic proteins (Bax, caspase-3, Cleaved caspase-3 and cytochrome C) were significantly increased while the expression level of antiapoptotic protein $(\mathrm{Bcl}-2)$ was significantly reduced $(p<$ $0.05, t$-test, Figure 8). It was observed that the expressions 

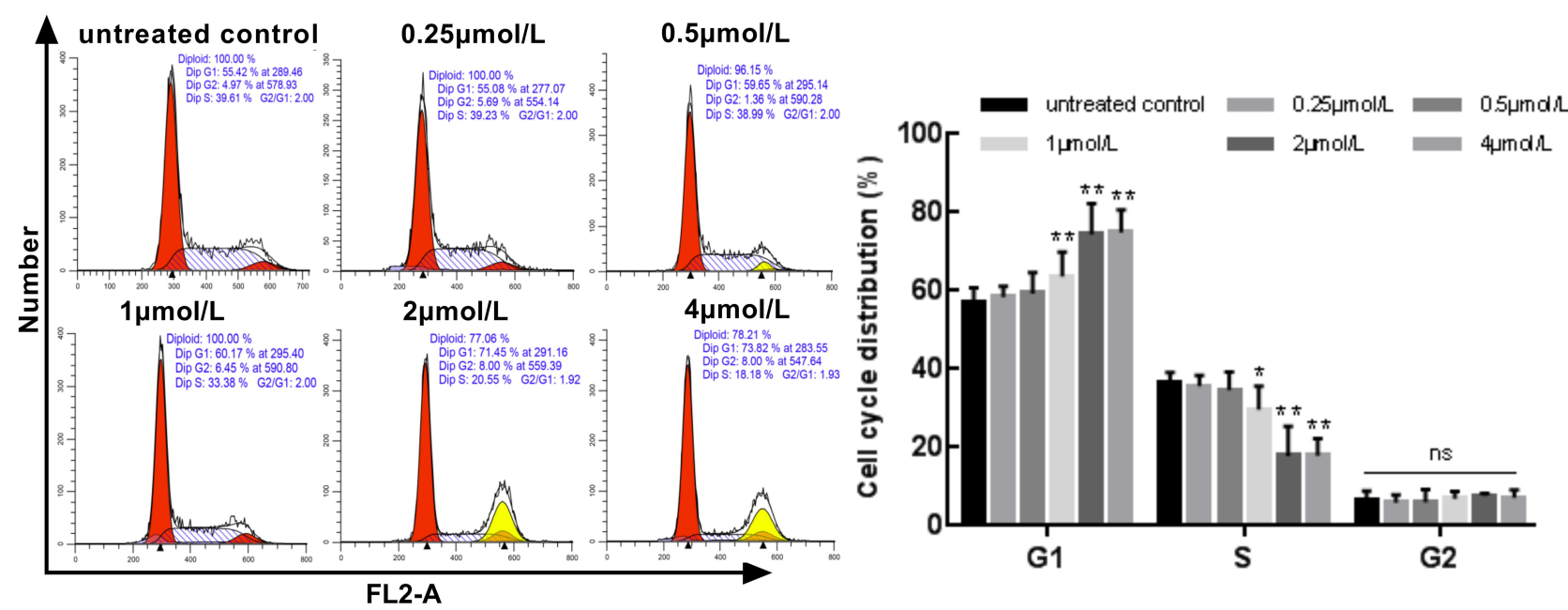

Figure 4 The effect of gracillin on the cell cycle of A549 cells. The proportion of cell number corresponding to cell cycle (GI phase, G2 phase and S phase) in each group after treatment with different concentrations of gracillin was demonstrated. The result of flow cytometry was on the left and the corresponding statistical chart was on the right. Data were expressed as mean $\pm \mathrm{SD}$. The statistical methods were $t$-test (GI phase and $\mathrm{S}$ phase) and ANOVA test (G2 phase). ${ }^{*} p<0.05$, $* * p<0.0 \mathrm{I}$, versus the untreated control group. Non-significant (NS) difference was observed in G2 phase between each group $(p>0.05)$.
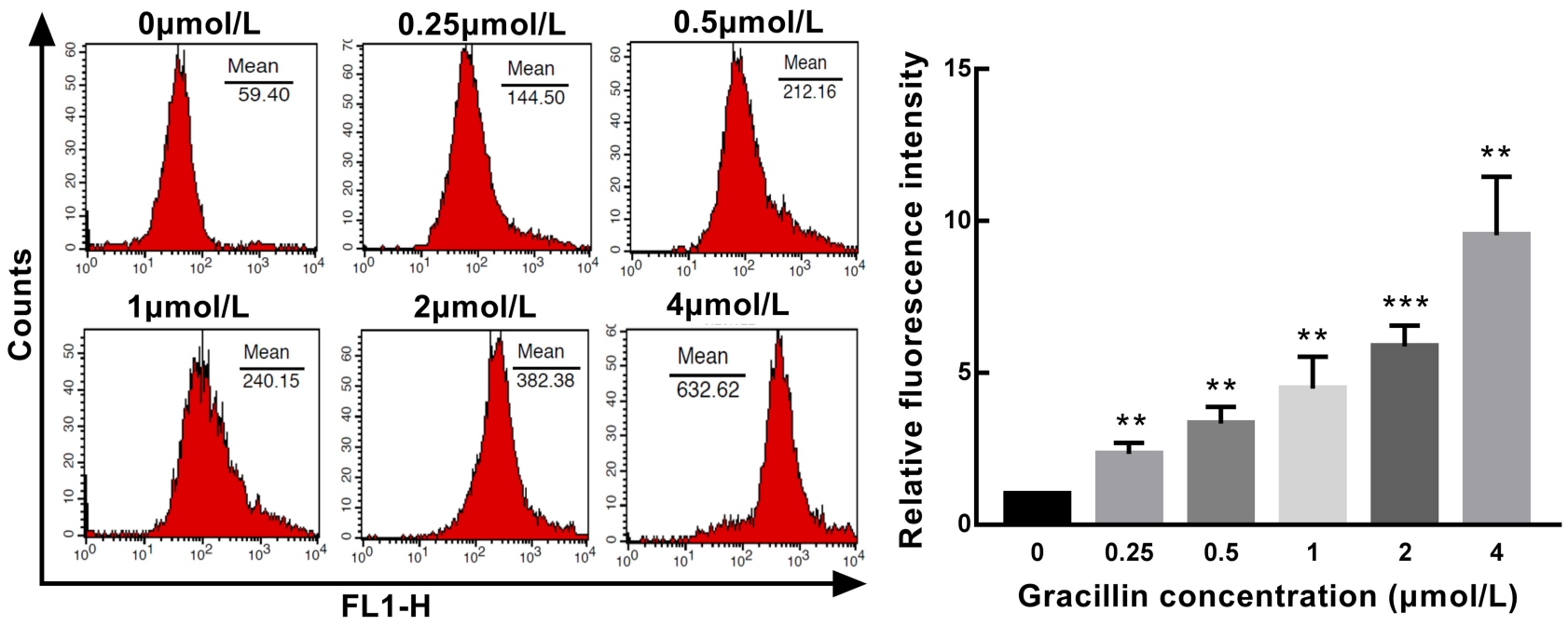

Figure 5 The concentration of intracellular calcium in A549 cells induced by gracillin. The relative level of intracellular calcium was shown as the ratio of the fluorescence intensity of the treated group to the blank group. The result of flow cytometry was on the left and the corresponding statistical chart was on the right. Data were expressed as mean $\pm S D$. T-test was used for the statistical analysis of results. $*^{*} p<0.01$, $* * * p<0.001$, versus the untreated control group.

of several apoptosis related genes and proteins of A549 cells were affected by gracillin.

\section{Discussion}

Recently, many natural products have been proved to have antitumor effects; the anti-tumor mechanism of the natural products includes direct killing, inhibiting the proliferation of cancer cells, promoting their apoptosis, and improving the immune function of the human body. ${ }^{24,25}$ In addition, previous studies show that these natural products can induce apoptosis of cancer cells via the mitochondrial pathway, such as polysaccharides ${ }^{26}$ and curcumin. ${ }^{27}$
After our research, we found that gracillin has great potential antiproliferative activity on A549 cells in vitro. So, we analyzed many key factors which related to the mitochondrial pathway of apoptosis.

According to the result of the cell proliferation inhibition experiment, the cytotoxic activity of gracillin on A549 cells has been proven. The cells treated by gracillin showed typical morphological changes of apoptosis. Then, the results of flow cytometry revealed that the apoptosis rate rose with the increase of gracillin concentration, which further confirmed that gracillin can significantly induce apoptosis of A549 cells. We analyzed the 

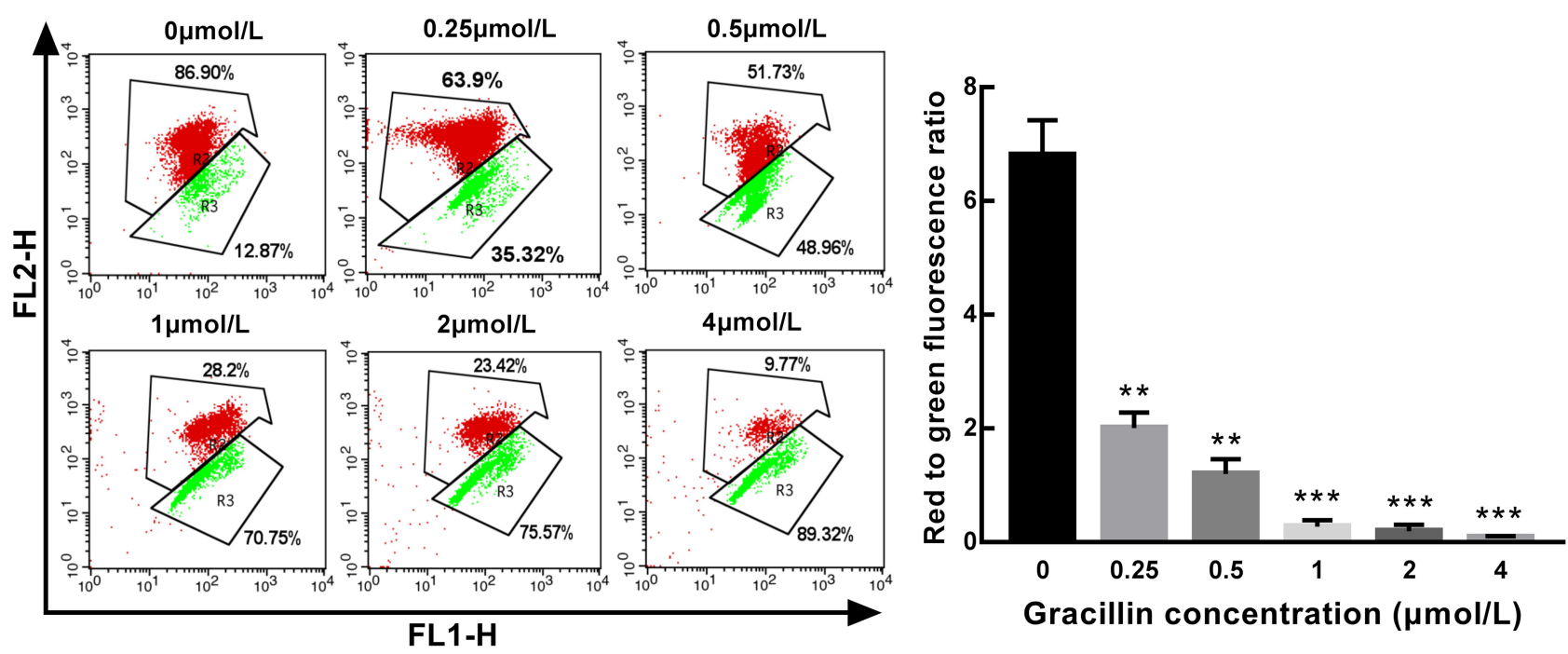

Figure 6 The effect of gracillin on mitochondrial membrane potential of A549 cells. The mitochondrial membrane potential was presented as the ratio of the red fluorescence intensity to the green fluorescence intensity. The result of flow cytometry was on the left and the corresponding statistical chart was on the right. Data were expressed as mean \pm SD. T-test was used for the statistical analysis of the data. $*^{*} p<0.01$, ***p $<0.001$, versus the untreated control group.
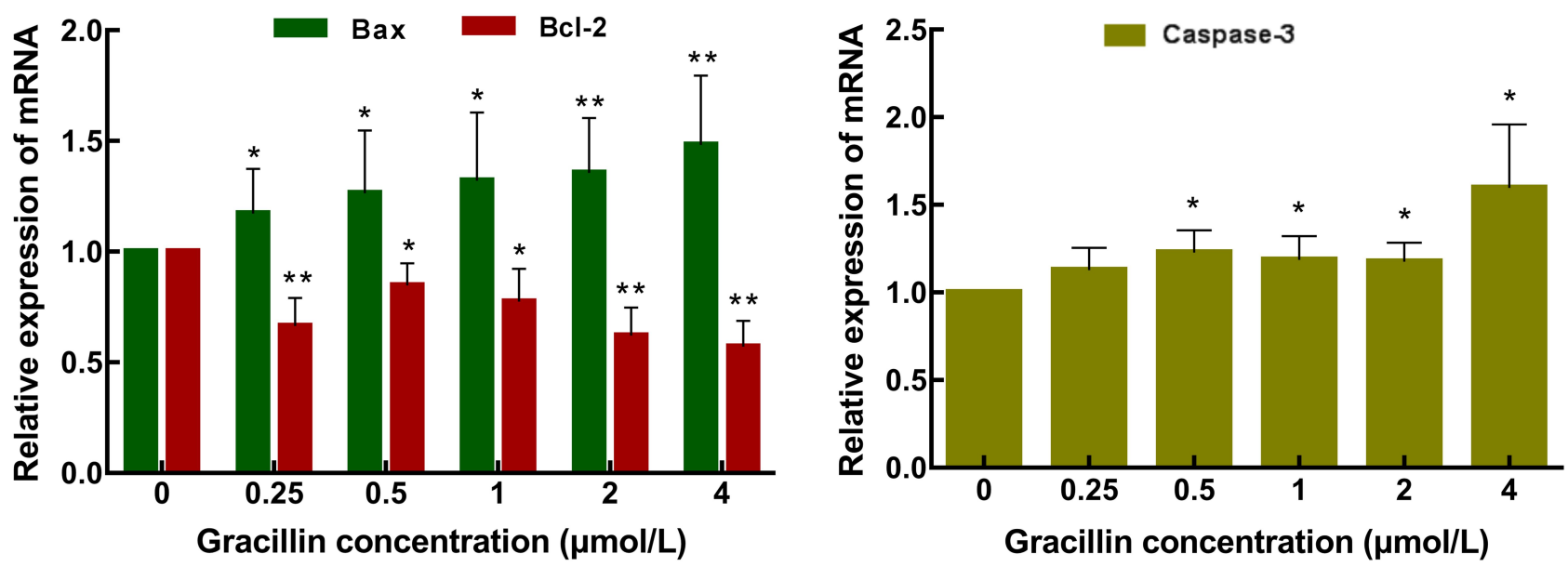

Figure 7 Analysis of expression levels of apoptosis-related genes. Real-time quantitative PCR was used to analyze the effect of gracillin on the mRNA expression level of $\mathrm{Bax}, \mathrm{Bcl}-2$ and caspase-3 in A549 cells. Data were expressed as mean \pm SD. T-test was used for the statistical analysis. ${ }^{*} p<0.05$, $* * p<0.01$, versus the untreated control group.

distribution of A549 cells in cell cycle after gracillin treatment, and found that the proportion of cells in G1 phase increased significantly, while that in $S$ phase decreased distinctly. Therefore, gracillin can induce cells cycle arrest in G1 phase; it indicates that gracillin can inhibit A549 cell proliferation and induce apoptosis by interfering with the process of the A549 cell cycle.

Intracellular calcium, as a second messenger, plays a crucial role in the process of cell apoptosis. Excessive release of calcium ions in the endoplasmic reticulum can induce changes in mitochondrial membrane permeability, ${ }^{28}$ which is beneficial to the opening of the outer mitochondrial membrane, thereby causing cell death. ${ }^{29,30}$ In this study, we tested the intracellular calcium concentration, and found that in the gracillin treatment group the concentration of calcium in A549 cells increased significantly, which verified that the intracellular calcium was involved in the apoptosis process of A549 cells induced by gracillin.

Mitochondria also play a vital role in cell survival and death, and participate in the mitochondrial pathway of cell apoptosis. The destruction of mitochondrial transmembrane potential is a common phenomenon in the process of apoptosis, and it is a sign of early apoptosis. ${ }^{31}$ A recent study $^{20}$ found that gracillin can destroy the biological function of mitochondria by inactivating SDH (succinate 
A

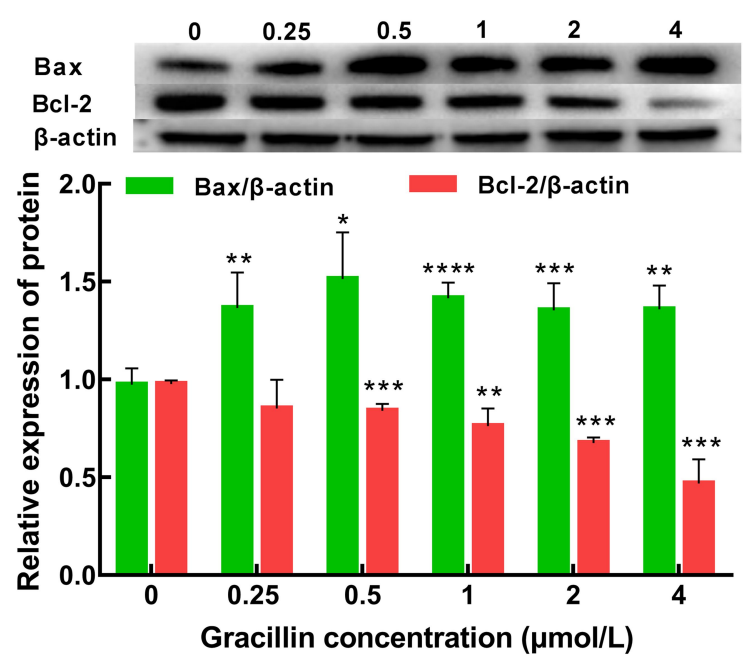

B

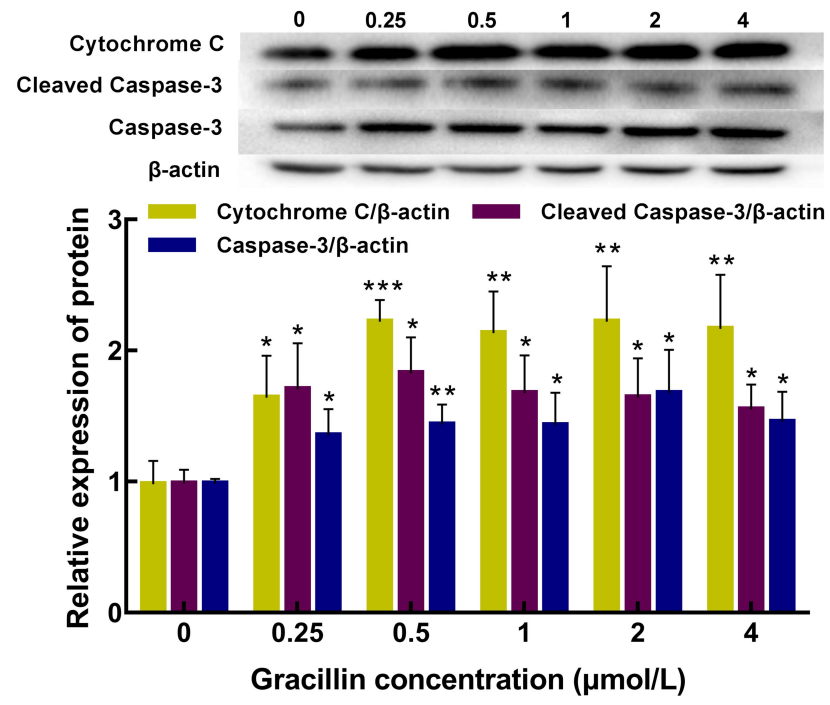

Figure 8 Analysis of expression levels of apoptosis-related proteins. (A) The expression of Bax and Bcl-2 proteins was determined by Western blotting. (B) The expression of caspase-3, cleaved caspase-3 and cytochrome $C$ proteins was determined by Western blotting. Data were expressed as mean \pm SD. T-test was used for the statistical analysis. $*_{p}<0.05, *_{p}<0.01,{ }^{* * *} p<0.001$, $*^{* * * *} p<0.000$ I, versus the untreated control group.

dehydrogenase), resulting in the decrease of mitochondrial membrane potential, therefore promoting the apoptosis of cancer cells. Likewise, we detected the changes of mitochondrial membrane potential, and the results showed that the mitochondrial membrane potential of A549 cells decreased with the increase of gracillin concentration. It confirmed that gracillin can destroy mitochondrial membrane potential and induce apoptosis of A549 cells.

At the same time, the apoptosis still depends on the interaction between Bcl-2 family proteins. ${ }^{32}$ In our study, with the treatment of gracillin, the expression of Bcl-2 decreased remarkably, with the concomitant increasing expression of Bax. Therefore, the increasing ratio of Bax to $\mathrm{Bcl}-2$ indicates that gracillin can induce the regulation of the Bcl-2 protein family to promote cell apoptosis. Additionally, cytochrome $\mathrm{C}$ is released after mitochondrial membrane potential is destroyed, and this process is considered as a key step to activate apoptosis. It is also essential for the activation of caspase cascade reactions in the mitochondrial pathway. ${ }^{11}$ Our results clearly verified that the expression of cytochrome $\mathrm{C}$ significantly increased after treatment with gracillin. Caspase-3, as an important executor in the process of apoptosis, has been found to be up-regulated in cancer cells by many natural products. $^{33}$ After receiving apoptosis signal stimulation, caspase- 3 is activated and converted to cleaved caspase3 , which is regarded as a reliable feature of apoptotic cells. $^{34}$ In this study, the data revealed that gracillin can significantly up-regulate the expression of cleaved caspase-3 in A549 cells.

Based on the above results, we speculate that gracillin promotes cell apoptosis by inducing the regulation of Bcl2 family proteins, which leads to the decrease of mitochondrial membrane potential and the release of apoptotic factors (cytochrome $\mathrm{C}$, calcium, etc.) in mitochondria, and then triggers the cascade reaction of the caspase family. This study suggests that the mechanism of gracillin inducing cell apoptosis may be related to the mitochondrial pathway. It should be noted that the antiproliferative effect of gracillin was verified in vitro on A549 cells, while in vivo elucidation needs to be further carried out.

\section{Conclusion}

This study confirmed that gracillin derived from Reineckia carnea has significant antiproliferative activity on A549 cells in vitro, and we further found that gracillin may induce apoptosis of A549 cells through the mitochondrial pathway. It exerts antiproliferative activity by regulating concentration of intracellular calcium, the mitochondrial membrane potential and the expression level of Bax, Bcl2, caspase-3, cleaved caspase-3, and cytochrome $\mathrm{C}$.

\section{Acknowledgments}

This work was supported by the National Natural Science Foundation of China (No. 31460082); the Jiangxi Natural Science Foundation (20181BAB205081); the Jiangxi 
Science and technology research project of the Department of Education (GJJ160982); the Open Project of Key Laboratory of Prevention and treatment of cardiovascular and cerebrovascular diseases, Ministry of Education (XN201809).

\section{Author Contributions}

All authors made a significant contribution to the work reported, whether that is in the conception, study design, execution, acquisition of data, analysis and interpretation, or in all these areas; took part in drafting, revising or critically reviewing the article; gave final approval of the version to be published; have agreed on the journal to which the article has been submitted; and agree to be accountable for all aspects of the work.

\section{Disclosure}

The authors declare that there are no conflicts of interests.

\section{References}

1. Ferlay J, Colombet M, Soerjomataram I, et al. Estimating the global cancer incidence and mortality in 2018: GLOBOCAN sources and methods. Int $J$ Cancer. 2019;144(8):1941-1953. doi:10.1002/ ijc. 31937

2. Torre LA, Bray F, Siegel RL, Ferlay J, Lortet-Tieulent J, Jemal A. Global cancer statistics, 2012. CA Cancer J Clin. 2015;65(2):87-108. doi:10.3322/caac. 21262

3. Zhou GW, Xiong Y, Chen S, Xia F, Li Q, Hu J. Anti-PD-1/PD-L1 antibody therapy for pretreated advanced nonsmall-cell lung cancer: A meta-analysis of randomized clinical trials. Medicine. 2016;95(35): e4611. doi:10.1097/MD.0000000000004611

4. Massarelli E, Papadimitrakopoulou V, Welsh J, Tang C, Tsao AS. Immunotherapy in lung cancer. Transl Lung Cancer Res. 2014;3 (1):53-63. doi:10.3978/j.issn.2218-6751.2014.01.01

5. Elmore S. Apoptosis: a review of programmed cell death. Toxicol Pathol. 2007;35(4):495-516. doi:10.1080/01926230701320337

6. D'Arcy MS. Cell death: a review of the major forms of apoptosis, necrosis and autophagy. Cell Biol Int. 2019;43(6):582-592. doi:10.1002/cbin. 11137

7. Pistritto G, Trisciuoglio D, Ceci C, Garufi A, D’Orazi G. Apoptosis as anticancer mechanism: function and dysfunction of its modulators and targeted therapeutic strategies. Aging. 2016;8(4):603-619. doi:10.18632/aging.100934

8. Kaczanowski S. Apoptosis: its origin, history, maintenance and the medical implications for cancer and aging. Phys Biol. 2016;13 (3):031001. doi:10.1088/1478-3975/13/3/031001

9. Goldar S, Khaniani MS, Derakhshan SM, Baradaran B. Molecular mechanisms of apoptosis and roles in cancer development and treatment. Asian Pac J Cancer Prev. 2015;16(6):2129-2144.

10. Wang X, Lu X, Zhu R, et al. Betulinic acid induces apoptosis in differentiated pc12 cells via ros-mediated mitochondrial pathway. Neurochem Res. 2017;42(4):1130-1140. doi:10.1007/s11064-0162147-y

11. Estaquier J, Vallette F, Vayssiere JL, Mignotte B. The mitochondrial pathways of apoptosis. Adv Exp Med Biol. 2012;942:157-183.

12. Knight T, Luedtke D, Edwards H, Taub JW, Ge Y. A delicate balance - The BCL-2 family and its role in apoptosis, oncogenesis, and cancer therapeutics. Biochem Pharmacol. 2019;162:250-261. doi:10.1016/j. bcp.2019.01.015
13. Zhao N, Wang H, Mu C, Wang Q, Cai Z. [Celastrol inhibits growth and increases apoptosis of human lung cancer A549 cells]. Xi Bao Yu Fen Zi Mian Yi Xue Za Zhi. 2018;34(12):1111-1115.Chinese.

14. Wang S, Hu Y, Yan Y, Cheng Z, Liu T. Sotetsuflavone inhibits proliferation and induces apoptosis of A549 cells through ROS-mediated mitochondrial-dependent pathway. BMC Complement Altern Med. 2018;18(1):235. doi:10.1186/s12906-0182300-z

15. Zhang ZQ, Chen JC, Zhou L, Qiu MH. Two new cholestane bisdesmosides from Reineckia carnea. Helv Chim Acta. 2009;90 (3):616-622. doi:10.1002/hlca.200790063

16. Fu XJ, Zou K, Zhang X, Cheng JF. Study on the anti-inflammatory effects and mechanisms of ethyl acetate extract of Reineckia carnea. Lishizhen Med Materia Medica Rese. 2013;24(4):822-825.

17. Zeng W, Bi YT. Antioxidant Activity of Five Extracts from Reineckea carnea to SH-SY5Y cell. Lishizhen Med Materia Medica Research. 2014;25(7):1549-1551.

18. Liu H, Yang JQ, Xiong L, Ma HM, Xu XJ. Preliminary study on the apoptosis mechanisms of lung cancer A549 cell induced by Reineckia carnea ethanol extracts. Chinese J Hospital Pharmacy. 2013;33(19):1580-1584.

19. Yang JQ, Ma HM, Liu H, Xu XJ, Wang Z, Liu Q. Study on in vitro anti-tumor activity of Reineckia carnea extract. $J$ Chinese Med Materials. 2013;36(4):618-621.

20. Min H-Y, Jang H-J, Park KH, et al. The natural compound gracillin exerts potent antitumor activity by targeting mitochondrial complex II. Cell Death Dis. 2019;10(11):810. doi:10.1038/s41419-019-2041-z

21. Chen C-R, Zhang J, Wu K-W, et al. Gracillin induces apoptosis in HL60 human leukemic cell line via oxidative stress and cell cycle arrest of G1. Die Pharmazie. 2015;70(3):199-204.

22. $\mathrm{Hu} \mathrm{K}$, Yao $\mathrm{X}$. The cytotoxicity of methyl protoneogracillin (NSC-698793) and gracillin (NSC-698787), two steroidal saponins from the rhizomes of Dioscorea collettii var. hypoglauca, against human cancer cells in vitro. Phytother Res. 2003;17(6):620-626. doi:10.1002/ptr.1211

23. Kang LP, Ma BP, Wang Y, Zhang J, Xiong CQ, Tang DW. Study on separation and identification of steroidal of Dioscorea nipponica Makino. Chinese Pharmaceutical J. 2005;(20):23-25.

24. Wang SF, Wu MY, Cai CZ, Li M, Lu JH. Autophagy modulators from traditional Chinese medicine: mechanisms and therapeutic potentials for cancer and neurodegenerative diseases. $J \quad$ Ethnopharmacol. 2016;194:861-876. doi:10.1016/j. jep.2016.10.069

25. Tao X, Yin L, Xu L, Peng J. Dioscin: A diverse acting natural compound with therapeutic potential in metabolic diseases, cancer, inflammation and infections. Pharmacol Res. 2018;137:259-269. doi:10.1016/j.phrs.2018.09.022

26. Pang G, Wang F, Zhang LW. Dose matters: direct killing or immunoregulatory effects of natural polysaccharides in cancer treatment. Carbohydr Polym. 2018;195:243-256. doi:10.1016/j. carbpol.2018.04.100

27. Langner E, Lemieszek MK, Rzeski W. Lycopene, sulforaphane, quercetin, and curcumin applied together show improved antiproliferative potential in colon cancer cells in vitro. $J$ Food Biochem. 2019;43(4):e12802. doi:10.1111/jfbc. 12802

28. Vercesi AE, Castilho RF, Kowaltowski AJ, et al. Mitochondrial calcium transport and the redox nature of the calcium-induced membrane permeability transition. Free Radic Biol Med. 2018;129:1-24. doi:10.1016/j.freeradbiomed.2018.08.034

29. Varghese E, Samuel SM, Sadiq Z, et al. Anti-cancer agents in proliferation and cell death: the calcium connection. Int J Mol Sci. 2019;20(12):3017. doi:10.3390/ijms20123017

30. La Rovere RM, Roest G, Bultynck G, Parys JB. Intracellular Ca(2+) signaling and $\mathrm{Ca}(2+)$ microdomains in the control of cell survival, apoptosis and autophagy. Cell Calcium. 2016;60(2):74-87. doi:10.1016/j.ceca.2016.04.005 
31. Christensen ME, Jansen ES, Sanchez W, Waterhouse NJ. Flow cytometry based assays for the measurement of apoptosis-associated mitochondrial membrane depolarisation and cytochrome c release. Methods. 2013;61(2):138-145. doi:10.1016/ j.ymeth.2013.03.020

32. Oberst A, Bender C, Green DR. Living with death: the evolution of the mitochondrial pathway of apoptosis in animals. Cell Death Differ. 2008;15(7):1139-1146. doi:10.1038/cdd.2008.65
33. Tian HY, Li ZX, Li HY, Wang HJ, Zhu XW, Dou ZH. Effects of 14 single herbs on the induction of caspase-3 in tumor cells: a brief review. Chin J Integr Med. 2013;19(8):636-640. doi:10.1007/ s11655-013-1539-y

34. Crowley LC, Waterhouse NJ. Detecting cleaved caspase-3 in apoptotic cells by flow cytometry. Cold Spring Harb Protoc. 2016;2016:11. doi:10.1101/pdb.prot087312

\section{Publish your work in this journal}

Drug Design, Development and Therapy is an international, peerreviewed open-access journal that spans the spectrum of drug design and development through to clinical applications. Clinical outcomes, patient safety, and programs for the development and effective, safe, and sustained use of medicines are a feature of the journal, which has also been accepted for indexing on PubMed Central. The manuscript management system is completely online and includes a very quick and fair peer-review system, which is all easy to use. Visit http://www. dovepress.com/testimonials.php to read real quotes from published authors. 